\title{
Growth hormone deficiency as a cause for short stature in Wiedemann-Steiner Syndrome
}

\author{
George Stoyle1,2, Siddharth Banka3,4, Claire Langley4, Elizabeth A Jones ${ }^{3,4}$ and Indraneel Banerjee \\ 'Department of Paediatric Endocrinology, Royal Manchester Children's Hospital, Manchester, UK, 2Manchester \\ Medical School, Faculty of Biology, Medicine and Health, University of Manchester, Manchester, UK, 3Manchester \\ Centre for Genomic Medicine, Division of Evolution \& Genomic Sciences, School of Biological Sciences, Faculty of \\ Biology, Medicine and Health, University of Manchester, Manchester, UK, and ${ }^{4}$ Manchester Centre for Genomic \\ Medicine, St Mary's Hospital, Manchester University, NHS Foundation Trust, Health Innovation Manchester, \\ Manchester, UK
}

\author{
Correspondence \\ should be addressed \\ to G Stoyle \\ Email \\ GStoyle1@gmail.com
}

\section{Summary}

Wiedemann-Steiner Syndrome (WSS) is a rare condition characterised by short stature, hypertrichosis of the elbow, intellectual disability and characteristic facial dysmorphism due to heterozygous loss of function mutations in KMT2A, a gene encoding a histone 3 lysine 4 methyltransferase. Children with WSS are often short and until recently, it had been assumed that short stature is an intrinsic part of the syndrome. GHD has recently been reported as part of the phenotypic spectrum of WSS. We describe the case of an 8-year-old boy with a novel heterozygous variant in KMT2A and features consistent with a diagnosis of WSS who also had growth hormone deficiency (GHD). GHD was diagnosed on dynamic function testing for growth hormone (GH) secretion, low insulin-like growth factor I (IGF-I) levels and pituitary-specific MRI demonstrating anterior pituitary hypoplasia and an ectopic posterior pituitary. Treatment with GH improved height performance with growth trajectory being normalised to the parental height range. Our case highlights the need for GH testing in children with WSS and short stature as treatment with GH improves growth trajectory.

\section{Learning points:}

- Growth hormone deficiency might be part of the phenotypic spectrum of Wiedemann-Steiner Syndrome (WSS).

- Investigation of pituitary function should be undertaken in children with WSS and short stature. A pituitary MR scan should be considered if there is biochemical evidence of growth hormone deficiency (GHD).

- Recombinant human growth hormone treatment should be considered for treatment of GHD.

\section{Background}

Wiedemann-Steiner Syndrome (WSS, OMIM\#605130) is a rare genetic disorder characterised by psychomotor delay, facial dysmorphism, hypertrichosis (especially of the elbow) and growth retardation $(1,2,3)$. WSS results from heterozygous loss of function KMT2A mutations (4). Recently, it has been reported that $50 \%$ of patients with WSS have growth hormone deficiency (GHD) (5). However, no details of endocrine investigations were provided in these patients. We report endocrine and imaging studies in a patient with genetically confirmed WSS and GHD and his response to therapy with GH.

\section{Case presentation}

Proband (8.6 years, male) was born to non-consanguineous parents of White European origin by normal delivery at 39 weeks, weighing $2.6 \mathrm{~kg}$ ( $-1.8 \mathrm{SDS}$ ) with evidence of intra-uterine growth restriction on antenatal ultrasound 
scanning. Vesico-ureteric reflux, mild hydronephrosis and hydroureter had been identified in early life. At 2.2 years of age, he presented with short stature (height: $75.5 \mathrm{~cm},-4.1 \mathrm{SDS}$ ), decreasing height velocity and poor weight gain (weight: $6.8 \mathrm{~kg},<-6.3$ SDS) (Fig. 1A). A review at 3 years of age showed that his height $(79.1 \mathrm{~cm}$ at age 3.0 years, -4.5 SDS) and weight $(9 \mathrm{~kg},-4.6 \mathrm{SDS})$ had remained suboptimal. His height was well below the mid-parental height $(174.0 \mathrm{~cm},-0.4 \mathrm{SDS})$. Investigations ruled out malabsorption. Although several allergies (soya and raw eggs) were noted, the coeliac screen was normal. Initial endocrine investigations showed low levels of the GH-dependent peptide IGF-I ( $20 \mu \mathrm{g} / \mathrm{L}$ (range: $28-247$ for age)) with other pituitary functions remaining normal. The cause for low IGF-I was attributed to low body weight instead of GHD.

Although the patient did not have obvious features of GHD other than short stature, growth hormone dynamic stimulation testing was performed as per local protocol. Glucagon-stimulated GH levels were low (basal: 1.8, peak: $3.3 \mu \mathrm{g} / \mathrm{L}$, expected values $>7 \mu \mathrm{g} / \mathrm{L}$ ), suggesting biochemical evidence for GHD. MR scanning of the pituitary and brain showed a hypoplastic anterior pituitary with an elongated posterior pituitary extending into the pituitary stalk (minor ectopic posterior pituitary), in keeping with a developmental abnormality of the pituitary gland (Fig. 1B). As the patient demonstrated an abnormal growth hormone response to stimulation and demonstrated radiological evidence of pituitary hypoplasia/ectopia, a second stimulation test was not performed in keeping with local protocol.

The patient was commenced on recombinant human GH treatment in a dose of $25 \mu \mathrm{g} / \mathrm{kg} / \mathrm{day}$, to which satisfactory height gain was achieved within the first year. While pre-treatment height velocity was $4.5 \mathrm{~cm} /$ year, post-treatment height velocity increased to $13.3 \mathrm{~cm} /$ year. Satisfactory height trajectory (height: $124.3 \mathrm{~cm}$ at 8.1 years, -0.8 SDS) was maintained in follow-up appointments with GH treatment administered in doses varying between 25 and $30 \mu \mathrm{g} / \mathrm{kg} /$ day. GHD continued to be isolated, with no evidence of wider pituitary dysfunction.

The patient was reviewed again at age 7.5 years; by this time, intellectual disability and an autistic spectrum disorder had been identified. Dysmorphism, including long eyelashes, thick arched eyebrows, downslanting and vertically narrow palpebral fissures, hypertelorism, low set ears, ptosis and mild facial asymmetry, was noted. The patient's DNA was analysed for a next generation sequencing (NGS)-based panel of 206 intellectual disability

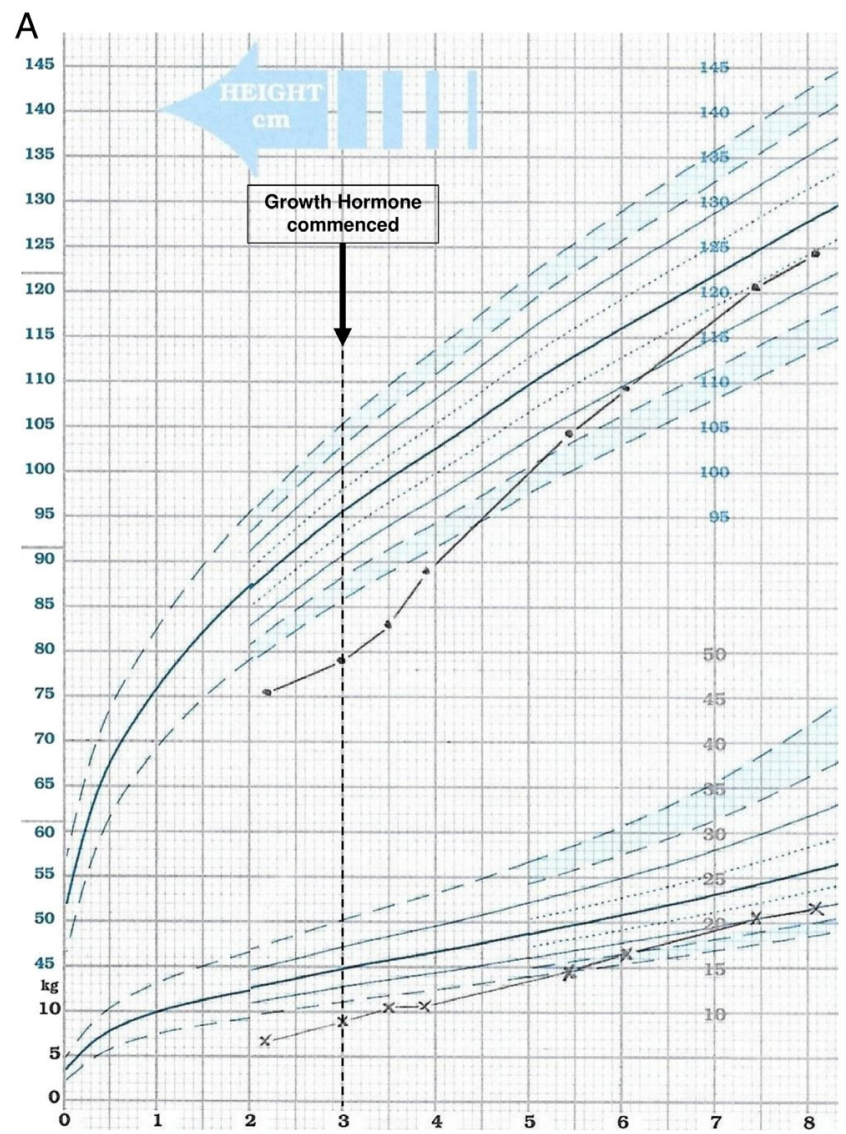

B

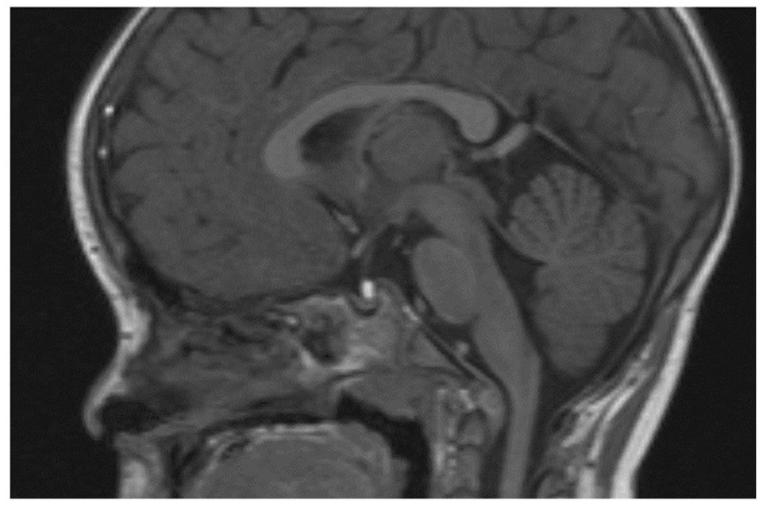

Figure 1

(A) Growth trajectory of patient with WSS demonstrating significant short stature prior to treatment and improvement in stature following treatment with growth hormone. (B) MR pituitary scan demonstrating hypoplastic anterior pituitary. The posterior pituitary is of abnormal configuration with elongation into the lower part of the pituitary stalk, suggesting minor ectopia. The combination of anterior pituitary hypoplasia and ectopic posterior pituitary is suggestive of abnormal pituitary development and explains biochemical evidence of GHD and short stature.

genes. A novel heterozygous KMT2A variant (c.7062delC p.(Ser2355LeufsTer18), RefSeq ID: NM_005933) predicted to be deleterious was identified. Reverse phenotyping 
confirmed that the phenotypic features were consistent with WSS (6). Targeted parental testing confirmed that the variant had arisen de novo.

\section{Discussion}

WSS is a rare genetic disease with variable phenotypes of presentation. Short stature is a recognised feature of WSS; the molecular basis for short stature has not been ascertained but had been assumed to be intrinsic to the genetic defect. A recent observational study suggested that GHD could be frequent in children with WSS (5). This study did not describe the methods by which GHD was diagnosed or report the response to GH treatment. As dynamic function testing has high false positive frequency, the validity of GHD diagnosis in WSS patients remains to be confirmed. In contrast, our case study has demonstrated auxological, biochemical and radiological evidence confirming GHD. Our findings suggest the possibility of a structural pituitary lesion in WSS causing GHD and short stature. Therefore, we would recommend thorough investigation of pituitary function in all children diagnosed with WSS, including a pituitary MR scan if there is biochemical evidence of GHD.

The combination of decelerating growth velocity, low GH to dynamic function testing, low IGF-I and MR scan findings of hypoplastic anterior pituitary/ ectopic posterior pituitary and satisfactory and sustained treatment response to $\mathrm{GH}$ are highly suggestive of a diagnosis of GHD. The possibility of GHD occurring as a chance association remains to be confirmed, although the observation of GHD in 50\% of WSS patients points towards a stronger and more intrinsic association (5). The latter is in contrast to the clinical experience in our centre where only one out of seven children with WSS had confirmed GHD.

The long-term outcome of GH treatment in patients with WSS has not been previously reported. In our patient, growth response was excellent to modest doses of GH treatment over a 6-year follow-up period. On regular monitoring, GH-dependent peptides IGF-I and IGFBP-3 remained within the normal range. No adverse events from $\mathrm{GH}$ administration were noted. There was no observed alteration in phenotypic characteristics of WSS. Our patient continues to be treated as any other child with GHD with strict monitoring for efficacy and adverse events arising from GH treatment; it remains to be seen if our patient develops wider pituitary dysfunction, experiences a pubertal growth spurt and if final height is appropriate for the parental height potential.
WSS is caused by KMT2A mutations (4). KMT2A encodes a histone 3 lysine 4 methyltransferase. Our observation indicates that KMT2A may have a role in pituitary development. Several other developmental disorders caused by mutations in histone lysine methyltransferases (KMTs) are known (7). Of these, KMT2D mutations that cause Kabuki syndrome (KS, OMIM\#147920) are notable. This is because GH deficiency has also been described in patients with KS $(8,9)$. CHD7 encodes a reader for H3K4 marks laid down by KMT2D (10). CHD7 mutations cause CHARGE syndrome (OMIM\#214800). Growth retardation is one of the cardinal features of CHARGE syndrome and GHD has also been described in patients with CHARGE (11).

We conclude that our case report illustrates the need to investigate pituitary function, in particular for $\mathrm{GH}$ secretion in all children diagnosed with WSS and short stature. Treatment with recombinant human GH could be considered as an option to improve height gain in patients with WSS and GHD.

\section{Declaration of interest}

The authors declare that there is no conflict of interest that could be perceived as prejudicing the impartiality of the research reported.

\section{Funding}

This work was supported by Research and Innovation at the Royal Manchester Children's Hospital.

\section{Patient consent}

Written informed consent from the patient's family was obtained for publication of the submitted article and accompanying images.

\section{Author contribution statement}

$\mathrm{G}$ Stoyle was responsible for the production of the written manuscript and accompanying figures. C Langley, S Banka and E A Jones were responsible for the genetic analysis and diagnosis of WSS and edited the manuscript. I Banerjee was the named physician who was responsible for conception, consent, production and overall editing of the manuscript.

\section{References}

1 Wiedemann HR, Kunze J, Grosse F-R \& Dibbern H. A syndrome of abnormal facies, short stature, and psychomotor retardation. In Atlas of Clinical Syndromes: A Visual Aid to Diagnosis for Clinicians and Practicing Physicians, 2nd ed., pp 198-199. London: Wolfe Publishing Ltd., 1989.

2 Steiner C \& Marques A. Growth deficiency, mental retardation and unusual facies. Clinical Dysmorphology 20009 155-156. (https://doi. org/10.1097/00019605-200009020-00021)

3 Min Ko J, Cho J, Yoo Y, Seo J, Choi M, Chae J, Lee H \& Cho T. Wiedemann-Steiner syndrome with 2 novel KMT2A mutations. 
Journal of Child Neurology 201632 237-242. (https://doi. org/10.1177/0883073816674095)

4 Jones W, Dafou D, McEntagart M, Woollard W, Elmslie F, HolderEspinasse M, Irving M, Saggar A, Smithson S, Trembath R, et al. De novo mutations in MLL cause Wiedemann-Steiner syndrome. American Journal of Human Genetics 201291 358-364. (https://doi. org/10.1016/j.ajhg.2012.06.008)

5 Baer S, Afenjar A, Smol T, Piton A, Gérard B, Alembik Y, Bienvenu T, Boursier G, Boute O, Colson C, et al. Wiedemann-Steiner syndrome as a major cause of syndromic intellectual disability: a study of 33 French cases. Clinical Genetics 201894 141-152. (https://doi. org/10.1111/cge.13254)

6 de Goede C, Yue W, Yan G, Ariyaratnam S, Chandler K, Downes L, Khan N, Mohan M, Lowe M \& Banka S. Role of reverse phenotyping in interpretation of next generation sequencing data and a review of INPP5E related disorders. European Journal of Paediatric Neurology 201620 286-295. (https://doi.org/10.1016/j.ejpn.2015.11.012)

7 Faundes V, Newman WG, Bernardini L, Canham N, ClaytonSmith J, Dallapiccola B, Davies SJ, Demos MK, Goldman A, Gill $\mathrm{H}$, et al. Histone lysine methylases and demethylases in the landscape of human developmental disorders. American Journal of Human Genetics 2018102 175-187. (https://doi.org/10.1016/j. ajhg.2017.11.013)

8 Banka S, Veeramachaneni R, Reardon W, Howard E, Bunstone S, Ragge N, Parker MJ, Crow YJ, Kerr B, Kingston H, et al. How genetically heterogeneous is Kabuki syndrome? MLL2 testing in 116 patients, review and analyses of mutation and phenotypic spectrum. European Journal of Human Genetics 201120 381-388. (https://doi. org/10.1038/ejhg.2011.220)

9 Schott D, Gerver W \& Stumpel C. Growth hormone therapy in children with Kabuki syndrome: 1-year treatment results. Hormone Research in Paediatrics 201788 258-264. (https://doi. org/10.1159/000479368)

10 Froimchuk E, Jang Y \& Ge K. Histone H3 lysine 4 methyltransferase KMT2D. Gene 2017627 337-342. (https://doi.org/10.1016/j. gene.2017.06.056)

11 Zentner G, Layman W, Martin D \& Scacheri P. Molecular and phenotypic aspects of CHD7 mutation in CHARGE syndrome. American Journal of Medical Genetics Part A 2010 152A 674-686. (https://doi.org/10.1002/ajmg.a.33323)

Received in final form 7 July 2018

Accepted 6 August 2018 